\title{
Approximate analytic solutions to non-symmetric stance trajectories of the passive Spring-Loaded Inverted Pendulum with damping
}

\author{
Uluc̣ Saranlı • Ömür Arslan • M. Mert Ankaralı • \\ Ömer Morgül
}

Received: 29 January 2010 / Accepted: 3 June 2010 / Published online: 9 July 2010

(C) Springer Science+Business Media B.V. 2010

\begin{abstract}
This paper introduces an accurate yet analytically simple approximation to the stance dynamics of the Spring-Loaded Inverted Pendulum (SLIP) model in the presence of non-negligible damping and non-symmetric stance trajectories. Since the SLIP model has long been established as an accurate descriptive model for running behaviors, its careful analysis is instrumental in the design of successful locomotion controllers. Unfortunately, none of the existing analytic methods in the literature explicitly take damping into account, resulting in degraded predictive accuracy when they are used for dissipative runners. We show that the methods we propose not only yield average predictive errors below $2 \%$ in the presence of
\end{abstract}

U. Saranlı ( $\varangle)$

Dept. of Computer Eng., Bilkent Univ., 06800 Bilkent, Ankara, Turkey

e-mail: saranli@cs.bilkent.edu.tr

Ö. Arslan · Ö. Morgül

Dept. of Electrical and Electronics Eng., Bilkent Univ., 06800 Bilkent, Ankara, Turkey

Ö. Arslan

e-mail: omur@ee.bilkent.edu.tr

Ö. Morgül

e-mail: morgul@ee.bilkent.edu.tr

M.M. Ankaralı

Dept. of Electrical and Electronics Eng., Middle East

Technical Univ., 06531 Ankara, Turkey

e-mail: ankarali@eee.metu.edu.tr significant damping, but also outperform existing alternatives to approximate the trajectories of a lossless model. Finally, we exploit both the predictive performance and analytic simplicity of our approximations in the design of a gait-level running controller, demonstrating their practical utility and performance benefits.

Keywords Legged locomotion · Hybrid dynamical systems · Spring-Loaded Inverted Pendulum . Analytic approximations · Damping · Gait control

\section{Introduction}

\subsection{Motivation and scope}

The adoption of mobile robots for tasks in unstructured outdoor environments has been slow due to the limited mobility and performance offered by existing wheeled and tracked platforms [11, 22]. In contrast, legged morphologies offer promising mobility advantages (as evidenced by numerous legged solutions devised by nature) and provide effective means with which power and actuator limitations can be overcome through the use of properly designed and tuned second order passive dynamics [2, 32]. However, the use of intuitive biological inspiration alone as a basis for both the design and control of such platforms has inherently limited promise since currently available sensor and actuator technologies are drastically different 
than their biological counterparts [13, 23]. In addition to such sources of inspiration, we need to have an accurate understanding of the physical principles underlying locomotory systems, while also developing analytical tools to provide a basis for the morphological design and control of legged robot platforms [18].

In this context, a very successful mathematical model for accurate prediction and control of legged locomotory behaviors has been the Spring-Loaded Inverted Pendulum (SLIP) model [35]. This model, consisting of a simple point mass riding on a single compliant leg, resulted from early studies in biomechanics $[2,3]$, revealing center of mass (COM) dynamics common to a surprisingly large range of biological runners with widely varying sizes and morphologies $[8,12]$. SLIP dynamics seem to elegantly capture the cyclic interchange between kinetic and potential energy that yields efficient and controllable locomotion, while providing a sufficiently simple analytical basis for a variety of questions related to the energetics, stability and control [16, 34].

The utility of the SLIP model is not limited to its descriptive power. A number of successful robotic platforms have been built, based either directly (e.g. Raibert's runners [28], the ARL monopods [1, 15], the Bow-Leg robot [37], the BiMasc platform [19] and the Jena-hopper) or indirectly (e.g. Scout quadrupeds [26], RHex [32] and Sprawl hexapods [10], BigDog [24] and others) on the principles embodied in this seemingly simple spring-mass model. Even though it has not yet been shown that neural control systems in running animals are organized in a way that internally encodes this model, its use as an explicit control target received considerable attention in the robotics community [25, 29], yielding both an intuitive high-level control interface for running behaviors, while also allowing a decomposition of the control problem into simpler pieces [30].

Given the almost universal dependence of existing literature related to legged robotic running on the SLIP model, there is a clear need for accurate tools for both the analysis and control of this model. Starting from earlier, intuitive approaches [27, 28, 33] to later formalizations [9, 14, 21, 36], a number of methods were developed to address the most significant problem with this seemingly simple model: its dynamics during phases of toe contact (i.e. stance) are non-integrable under the effect of gravity [17]. Available methods suffer from unrealistic assumptions such as the conservation of angular momentum and the neglection of damping losses. The former is readily violated in the presence of gravity with non-symmetric, transient strides [20], and the latter is an undesirable but unavoidable disturbance present in all physical legged platforms. In this paper, we propose a new analytical approximation to the trajectories of the SLIP model that is significantly more accurate in the presence of both passive damping and non-symmetric steps under gravity, yielding a critical analytical tool for both the design and control of dynamically stable legged platforms. We believe that the resulting tools are sufficiently accurate to support physical implementation of novel dexterous locomotion controllers on rough terrain such as those presented in [7].

\subsection{Contributions}

Our primary contribution in this paper is the derivation of a highly accurate analytical approximation to the stance map of a planar hopper with linear compliance and damping in the leg, with additional corrections introduced to compensate for the effect of gravity on the angular momentum for non-symmetric steps. The resulting analytic return map for running behaviors has substantial practical utility since it can be used as a basis for the design of locomotion controllers for physically plausible robot morphologies on rough terrain, while also providing an analytical tool for the characterization of associated dynamic legged behaviors. None of the existing alternatives in the literature explicitly take damping into account, making their direct application to such systems very difficult and inaccurate.

In order to illustrate the applicability and performance of our approximations in such settings, we carefully characterize their predictive performance with respect to a simulated model within a non-dimensional formulation, across a large range of initial conditions and parameter combinations. We compare our results with two previously available analytic approximation methods proposed in [14] and [36], first in the context of a lossless SLIP model for which they were designed for, and then a dissipative runner that challenges their underlying assumptions. Finally, we present how our approximations can be used to achieve high level control of legged locomotion by designing a deadbeat controller for the regulation of running speed and hopping height of a simulated planar monopod. Once again, we compare the performance of our proposed 
Table 1 State variables, parameters and the definitions of their dimensionless counterparts for the SLIP model. Variables with and without bars correspond to physical and dimensionless quantities, respectively

\begin{tabular}{llll}
\hline $\begin{array}{l}\text { Physical } \\
\text { quantity }\end{array}$ & $\begin{array}{l}\text { Dimensionless } \\
\text { group }\end{array}$ & Definition & Description \\
\hline $\bar{t}$ & $t$ & & \\
{$[\bar{y}, \bar{z}]$} & $\mathbf{b}:=[y, z]$ & $:=[\bar{t} / \lambda$ & Time (where $\left.\lambda:=\sqrt{l_{0} / g}\right)$ \\
{$[\bar{\rho}, \bar{\theta}]$} & $\mathbf{q}:=[\rho, \theta]$ & $:=\left[\bar{\rho} / l_{0}, \bar{\theta}\right]$ & Body position \\
$\bar{y}$ & $y_{f}$ & $:=\bar{y}_{f} / l_{0}$ & Leg length and leg angle \\
$k$ & $\kappa$ & Foot position \\
$d$ & $c$ & $\left.:=l_{0} /(m g)\right)$ & Leg spring stiffness \\
$\bar{F}$ & $F$ & $:=\bar{F} /(m g)$ & Leg viscous damping \\
$\bar{E}$ & $E$ & $:=\bar{E} /\left(m g l_{0}\right)$ & Force variables \\
$\bar{p}_{\bar{\theta}}$ & $p_{\theta}$ & $:=p_{\theta} /\left(\lambda /\left(m l_{0}^{2}\right)\right)$ & Energy variables \\
$\bar{p}_{\bar{\rho}}$ & $p_{\rho}$ & $:=\bar{p}_{\bar{\rho}}\left(\lambda /\left(m l_{0}\right)\right)$ & Angular momentum \\
\hline & & & Radial momentum \\
\hline
\end{tabular}

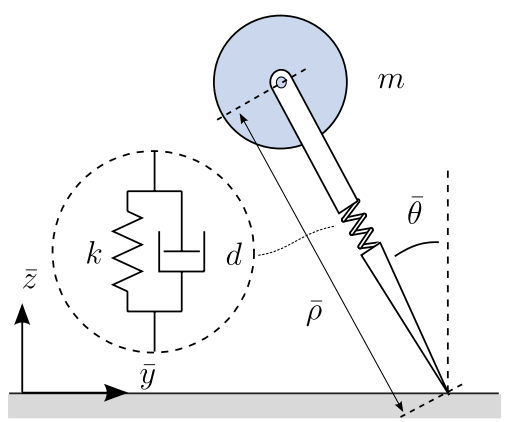

Fig. 1 The Spring-Loaded Inverted Pendulum model with damping

controller to a similar application of alternative approximations in the literature.

\section{The lossy Spring-Loaded Inverted Pendulum model}

\subsection{System model and dynamics}

Figure 1 shows the Spring-Loaded Inverted Pendulum model we use in this paper, consisting of a point mass $m$ attached to a freely rotating massless leg, endowed with a linear spring-damper pair of compliance $k$, rest length $l_{0}$ and, differently from the ideal SLIP model, viscous damping $d$. Throughout locomotion, the model alternates between stance and flight phases, further divided into the compression, decompression and ascent, descent subphases, respectively. Four important events define discrete transitions between these subphases: touchdown, bottom, liftoff, and apex. During flight, the body is assumed to be a projectile acted upon by gravity, whereas in stance, the toe is assumed to be fixed on the ground and the mass feels radial leg forces. Table 1 details all relevant variables and parameters for this model.

In order to eliminate redundant parameters and provide an efficient way to interpret our simulation results, we will use a dimensionless formulation. Redefining time as $t:=\bar{t} / \lambda$ with $\lambda:=\sqrt{l_{0} / g}$, scaling all distances with the spring rest length $l_{0}$, dimensionless SLIP dynamics are given as

Flight: $\left[\begin{array}{l}\ddot{y} \\ \ddot{z}\end{array}\right]=\left[\begin{array}{c}0 \\ -1\end{array}\right]$,

Stance: $\left[\begin{array}{c}\ddot{\rho} \\ \ddot{\theta}\end{array}\right]=\left[\begin{array}{c}\rho \dot{\theta}^{2}-\kappa(\rho-1)-c \dot{\rho}-\cos \theta \\ (-2 \dot{\rho} \dot{\theta}+\sin \theta) / \rho\end{array}\right]$,

with flight dynamics written in Cartesian coordinates and stance dynamics in polar coordinates for convenience. Transformations between these coordinate systems require the foot location $y_{f}$ as a separate state which undergoes discrete changes from touchdown to touchdown. Note, also, that $(d / d t)^{n}=\lambda^{n}(d / d \bar{t})^{n}$ and all time derivatives are with respect to the newly defined, scaled time variable. Throughout the rest of the paper, we will only work with dimensionless quantities and hence will not explicitly mention their dimensionless nature unless necessary.

\subsection{Modeling of running gaits: the apex return map}

A commonly used and convenient abstraction for both the analysis and control of the SLIP model is the apex 


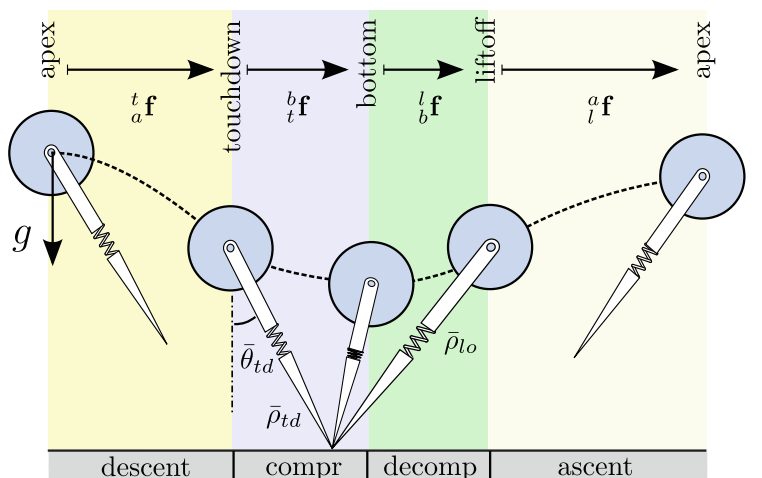

Fig. 2 SLIP locomotion phases and associated return map components

return map, defined as the Poincaré section taken at $\dot{z}=0$ during flight [35], leading to the definition of the apex state as

$X_{a}:=\left[y_{a}, z_{a}, \dot{y}_{a}\right]^{T}$.

Such a section not only reduces the dimension of the system, but also allows a convenient discrete, tasklevel abstraction suitable for the characterization of steady-state gaits [35], designing controllers [31] and analyzing their stability [4]. In this paper, we also adopt the apex return map for both evaluating the performance of our approximations and designing gait controllers based on these approximations.

The apex return map for the SLIP model is a combination of four subsequent maps, illustrated in Fig. 2, corresponding to descent (apex to touchdown), compression (touchdown to bottom), decompression (bottom to liftoff) and ascent (liftoff to apex) subphases of locomotion, denoted by ${ }_{a}^{t} \mathbf{f},{ }_{t}^{b} \mathbf{f},{ }_{b}^{l} \mathbf{f}$ and ${ }_{l}^{a} \mathbf{f}$, respectively. The apex return map hence takes the form

$$
\begin{aligned}
X_{a}^{k+1} & ={ }_{a}^{a} \mathbf{f}_{\left[\theta_{t d}, \rho_{t d}, \rho_{l o}, \kappa_{c}, \kappa_{d}\right]}\left(X_{a}^{k}\right) \\
& :=\left({ }_{l}^{a} \mathbf{f} \circ{ }_{b}^{l} \mathbf{f}_{\left[\kappa_{d}, \rho_{l o}\right]} \circ{ }_{t}^{b} \mathbf{f}_{\left[\kappa_{c}\right]} \circ{ }_{a}^{t} \mathbf{f}_{\left[\theta_{t d}, \rho_{t d}\right]}\right)\left(X_{a}^{k}\right),
\end{aligned}
$$

where several key parameters that can be used to control progression through these submaps are explicitly shown. In particular, $\theta_{t d}, \kappa_{c}$ and $\kappa_{d}$ denote the familiar touchdown angle, compression and decompression spring constants as in many earlier hopper implementations [1,28], while $\rho_{t d}$ and $\rho_{l o}$ denote leg lengths at touchdown and liftoff similarly to control parameters used by the Bow-Leg hopper [37]. All components of the return map, together with relevant control inputs, are illustrated in Fig. 2.
It is important to note that from among these available control inputs, any choice of three that includes the touchdown angle $\theta_{t d}$ grants full controllability to the system (i.e. gives independent authority over all of the apex states) [35, 37], with the primary difference being in the way the energy of the system is regulated. In this paper, we will assume that the landing and liftoff leg lengths are explicitly controllable as in the Bow-Leg hopper.

Given this choice, the descent and ascent submaps are

$$
\begin{aligned}
X_{t d}^{k} & ={ }_{a}^{t} \mathbf{f}\left(X_{a}^{k}\right) \\
& :=\left[y_{a}+\dot{y}_{a} \Delta t_{d}, \rho_{t d} \cos \theta_{t d}, \dot{y}_{a},-\Delta t_{d}\right], \\
X_{a}^{k+1} & ={ }_{l}^{a} \mathbf{f}\left(X_{l o}^{k}\right) \\
& :=\left[y_{l}+\dot{y}_{l} \Delta t_{a}, \rho_{l o} \cos \theta_{l o}+\dot{z}_{l}^{2} / 3, \dot{y}_{l}\right],
\end{aligned}
$$

where we define the touchdown and liftoff states as $X_{t d}:=\left[\rho_{t d}, \theta_{t d}, \dot{\rho}_{t d}, \dot{\theta}_{t d},\right]^{T}$ and $X_{l o}:=$ $\left[\rho_{l o}, \theta_{l o}, \dot{\rho}_{l o}, \dot{\theta}_{l o},\right]^{T}$, respectively, with $\Delta t_{d}:=$ $\sqrt{2\left(z_{a}-\rho_{t d} \cos \theta_{t d}\right)}$. Note that both $X_{t d}$ and $X_{l o}$ are defined as intermediate states and hence incorporate additional, redundant dimensions for convenience as compared to the three-dimensional apex states in (3).

Not surprisingly, the most difficult components in the apex return map are the compression and decompression phases, both requiring closed-form integration of the stance dynamics. While there are a number of existing approximations for this purpose, none of them incorporate damping and have substantial difficulty in modeling the effect of gravity on the angular momentum in the presence of stance trajectories that are not symmetric with respect to the vertical.

\subsection{Existing analytical tools for the undamped SLIP}

In the following sections, we review two important analytical approximations to the stance dynamics of the undamped SLIP model. Our approximation is inspired from the method proposed in [14], but substantially improves predictive and control performance by accurately incorporating the effects of damping and varying angular momentum during stance.

\subsubsection{Iterative approximate stance map by Schwind et al.}

In [36], Schwind uses an iterative application of the mean-value theorem for integral operators to obtain an 
analytical approximation to the stance dynamics of a lossless SLIP. Their derivation is based on a Hamiltonian formulation of the conservative SLIP dynamics, yielding the dimensionless Hamiltonian function as

$$
H:=\frac{1}{2}\left(p_{\rho}^{2}+\frac{p_{\theta}^{2}}{\rho^{2}}\right)+\frac{1}{2} \kappa(1-\rho)^{2}+\rho \cos \theta .
$$

The equations of motion can then be written in terms of the radial degree of freedom as an independent variable by assuming that the system energy stays constant, and solving the equation $H\left(p_{\rho}\right)=E$ to yield $H^{\dagger}:=H^{-1}(E)=p_{\rho}$ as a function of the leg length $\rho$. It then becomes possible to obtain an approximate solution, yielding the following solution for the decompression phase:

$$
\begin{aligned}
& \hat{t}_{d_{(n+1)}}(\rho)=t_{b}+\left(\rho-\rho_{b}\right) / H_{n}^{\dagger}, \\
& \hat{\theta}_{(n+1)}(\rho)=\theta_{b}+\hat{p}_{\theta}(\hat{\xi})\left(\rho-\rho_{b}\right) /\left(\hat{\xi}^{2} H_{n}^{\dagger}\right), \\
& \hat{p}_{\theta(n+1)}(\rho)=p_{\theta b}+\hat{\xi} \sin \left(\hat{\theta}_{n}(\hat{\xi})\right)\left(\rho-\rho_{b}\right) / H_{n}^{\dagger}, \\
& \hat{p}_{\rho_{(n+1)}}(\rho)=H_{n+1}^{\dagger},
\end{aligned}
$$

where $n$ indicates the iteration number, $\hat{\xi}:=3 \rho_{b} / 4+$ $\rho / 4$ arises from the application of the mean value theorem, and $t_{b}, \rho_{b}, \theta_{b}$ and $p_{\theta b}$ represent the system state at bottom.

Given touchdown states, $t_{t d}, \rho_{t d}, \theta_{t d}$ and $p_{\theta t d}$, the compression phase mapping can be similarly derived as

$$
\begin{aligned}
& \hat{t}_{c_{(n+1)}}(\rho)=t_{t d}-\left(\rho-\rho_{t d}\right) / H_{n}^{\dagger}, \\
& \hat{\theta}_{(n+1)}(\rho)=\theta_{t d}-\hat{p}_{\theta n}(\hat{\xi})\left(\rho-\rho_{t d}\right) /\left(\hat{\xi}^{2} H_{n}^{\dagger}\right), \\
& \hat{p}_{\theta(n+1)}(\rho) \\
& \quad=p_{\theta t d}-\hat{\xi} \sin \left(\hat{\theta}_{n}(\hat{\xi})\right)\left(\rho-\rho_{t d}\right) / H_{n}^{\dagger}, \\
& \hat{p}_{\rho_{(n+1)}}(\rho)=-H_{n+1}^{\dagger},
\end{aligned}
$$

where $\hat{\xi}:=3 \rho_{t d} / 4+\rho / 4$.

Furthermore, these equations can be iterated to yield increasingly accurate analytic approximations. However, since the solutions are formulated as a function of the radial state, finding the bottom instant represents one of the problems with this approach. Nevertheless, it is possible to use an energy-based solution to the bottom radial length $[6,29]$. We omit the details of this derivation for space considerations.
It is important to note that Schwind's method critically relies on the assumption of a lossless plant model and conservation of energy, making its direct application to a lossy system very difficult, requiring nontrivial modifications. Furthermore, its analytical complexity substantially increases with each iteration, at least two of which are required for reasonably accurate results.

\subsubsection{Simple approximate stance map by Geyer et al.}

In [14], Geyer proposes a method to obtain an analytical approximation to the stance dynamics of a lossless SLIP. In this section we review their method, adapted to use the leg length control parameters $\rho_{t d}$ and $\rho_{l o}$ within a dimensionless formulation compatible to ours.

As proposed in [14], if we assume that the stance phase is predominantly vertical with a sufficiently small angular span $\Delta \theta$, the effect of gravity can be linearized around $\theta=0$, making both the angular momentum $p_{\theta}$ and the total mechanical energy constants of motion. Combined with the assumption that the relative spring compression remains sufficiently small with $|1-\rho| \ll 1$, and some additional approximations detailed in [14], analytic expressions for the radial and angular stance trajectories can be found as

$$
\begin{aligned}
\rho(t)= & 1+a+b \sin \left(\hat{\omega}_{0} t\right), \\
\theta(t)= & \theta_{t d}+p_{\theta}(1-2 a)\left(t-t_{t d}\right) \\
& +\frac{2 b p_{\theta}}{\hat{\omega}_{0}}\left[\cos \left(\hat{\omega}_{0} t\right)-\cos \left(\hat{\omega}_{0} t_{t d}\right)\right]
\end{aligned}
$$

in dimensionless coordinates with the definitions

$$
\begin{aligned}
\hat{\omega}_{0} & :=\sqrt{\kappa+3 p_{\theta}^{2}}, \\
a & :=\left(p_{\theta}^{2}-1\right) / \hat{\omega}_{0}^{2}, \\
b & :=\sqrt{a^{2}+\left(2 E-p_{\theta}^{2}-2\right) / \hat{\omega}_{0}^{2}},
\end{aligned}
$$

where the total mechanical energy, denoted by $E$, is computed based on prior apex states. Subsequently, leg length control inputs at touchdown and liftoff can be used as boundary conditions on (16) to determine touchdown, bottom and liftoff times relative to an arbitrary time origin as

$$
\begin{aligned}
& t_{t d}=\left(\pi-\arcsin \left(\left(\rho_{t d}-1-a\right) / b\right)\right) / \hat{\omega}_{0}, \\
& t_{l o}=\left(2 \pi+\arcsin \left(\left(\rho_{l o}-1-a\right) / b\right)\right) / \hat{\omega}_{0},
\end{aligned}
$$




$$
t_{b}=3 \pi /\left(2 \hat{\omega}_{0}\right)
$$

Following a final, energy-based correction on the horizontal component of the liftoff velocity, these derivations yield an analytically simple but accurate approximation to the symmetric stance trajectories of a lossless SLIP.

Unfortunately, both assumptions in these derivations, the conservation of angular momentum and the lack of any damping, limit their direct applicability to the control of maneuverable running on practical legged robots. Nevertheless, as described in Sect. 3, we will be able to adapt key ideas from this method in the derivation of our new approximations with substantially more general applicability.

\section{A new analytic approximation to the stance map}

We start the presentation of our approximations by derivations based on assuming conservation of angular momentum in Sect. 3.1, followed in Sect. 3.2 by the computation of components necessary to assemble the apex return map and conclude in Sect. 3.3 with a method to reintroduce gravity and compensate for inaccuracies resulting from our starting assumption.

\subsection{Approximating stance trajectories under damping}

We first rearrange the angular component of (2) to yield a more convenient form of the stance dynamics as

$$
\begin{aligned}
& \ddot{\rho}=\rho \dot{\theta}^{2}-\kappa(\rho-1)-c \dot{\rho}-\cos \theta, \\
& 0=\frac{d}{d t}\left(\rho^{2} \dot{\theta}\right)-\rho \sin \theta .
\end{aligned}
$$

In order to derive our analytical approximation, we continue with the commonly used assumption that the leg remains close to the vertical throughout the entire stance phase. Consequently, as in [14], the gravitational potential can be linearized around $\theta=0$. Note that this assumption, as noted before, is violated for non-symmetric stance trajectories that arise during transient locomotion steps. However, as we describe in Sect. 3.3, it will be possible to introduce an explicit correction to the angular momentum by separately considering the effects of gravity. Nevertheless, for now, the resulting conservation of the angular momentum $p_{\theta}:=\rho^{2} \dot{\theta}$ reduces the radial dynamics of (24) to

$\ddot{\rho}+c \dot{\rho}+\kappa \rho-p_{\theta}^{2} / \rho^{3}=-1+\kappa$.

Unfortunately, even these reduced dynamics do not admit an analytical solution. However, using the method proposed by Geyer [14], we further assume that the relative spring compression remains sufficiently small with $|1-\rho| \ll 1$, allowing the term $1 / \rho^{3}$ to be approximated by a Taylor series expansion around $\rho=1$ to yield

$1 /\left.\rho^{3}\right|_{\rho=1} \approx 1-3(\rho-1)+O\left((\rho-1)^{2}\right)$.

This assumption remains valid as long as the leg compression during stance is not excessive (i.e. not more that $75 \%$ of the leg rest length), which is true for most running behaviors except extreme cases such as kangaroo hopping or quadrupedal pronking behaviors. Nevertheless, under this approximation, (26) reduces to

$\ddot{\rho}+c \dot{\rho}+\left(\kappa+3 p_{\theta}^{2}\right) \rho=-1+\kappa+4 p_{\theta}^{2}$,

where we define the natural frequency of the system, $\hat{\omega}_{0}:=\sqrt{\kappa+3 p_{\theta}^{2}}$, the damping ratio, $\xi:=c /\left(2 \hat{\omega}_{0}\right)$, the damped frequency, $\omega_{d}:=\hat{\omega}_{0} \sqrt{1-\xi^{2}}$, and the forcing term, $F:=-1+\kappa+4 p_{\theta}^{2}$, and obtain

$\ddot{\rho}+2 \xi \hat{\omega}_{0} \dot{\rho}+\hat{\omega}_{0}^{2} \rho=F$.

This is a second-order ordinary differential equation that can easily be solved analytically. Assuming $\xi<1$, we have

$$
\begin{aligned}
\rho(t)= & e^{-\xi \hat{\omega}_{0} t}\left(A \cos \left(\omega_{d} t\right)+B \sin \left(\omega_{d} t\right)\right) \\
& +F / \hat{\omega}_{0}^{2}
\end{aligned}
$$

with $A$ and $B$ determined by touchdown states as

$$
\begin{aligned}
& A=\rho_{t d}-F / \hat{\omega}_{0}^{2}, \\
& B=\left(\dot{\rho}_{t d}+\xi \hat{\omega}_{0} A\right) / \omega_{d} .
\end{aligned}
$$

Simple differentiation yields the radial velocity as

$$
\begin{aligned}
\dot{\rho}(t)= & -M e^{-\xi \hat{\omega}_{0} t}\left(\xi \hat{\omega}_{0} \cos \left(\omega_{d} t+\phi\right)\right. \\
& \left.+\omega_{d} \sin \left(\omega_{d} t+\phi\right)\right),
\end{aligned}
$$


where $M:=\sqrt{A^{2}+B^{2}}$ and $\phi:=\arctan (-B / A)$. Further manipulations yield the simplest form of the radial motion as

$\rho(t)=M e^{-\xi \hat{\omega}_{0} t} \cos \left(\omega_{d} t+\phi\right)+F / \hat{\omega}_{0}^{2}$,

$\dot{\rho}(t)=-M \hat{\omega}_{0} e^{-\xi \hat{\omega}_{0} t} \cos \left(\omega_{d} t+\phi+\phi_{2}\right)$,

where $\phi_{2}:=\arctan \left(-\sqrt{1-\xi^{2}} / \xi\right)$.

Now that an analytical approximation to the radial trajectory is available, the angular trajectory can be determined by using the constancy of the angular momentum $\dot{\theta}=p_{\theta} / \rho^{2}$. Linearizing $1 / \rho^{2}$ around $\rho=1$ yields

$1 /\left.\rho^{2}\right|_{\rho=1}=1-2(\rho-1)+O\left((\rho-1)^{2}\right)$,

with which we can obtain an analytical solution for the angular velocity of the leg as

$\dot{\theta}(t)=3 p_{\theta}-2 p_{\theta} F / \hat{\omega}_{0}^{2}-2 p_{\theta} M e^{-\xi \hat{\omega}_{0} t} \cos \left(\omega_{d} t+\phi\right)$.

Integration then yields the angular trajectory of the leg as

$$
\begin{aligned}
\theta(t)= & \theta_{t d}+X t+Y\left(e^{-\xi \hat{\omega}_{0} t} \cos \left(\omega_{d} t+\phi-\phi_{2}\right)\right. \\
& \left.-\cos \left(\phi-\phi_{2}\right)\right),
\end{aligned}
$$

where $X:=3 p_{\theta}-2 p_{\theta} F / \hat{\omega}_{0}^{2}$ and $Y:=2 p_{\theta} M / \hat{\omega}_{0}$.

The approximate solutions in (33), (34), (37) and (36) provide a sufficiently simple analytic solution to the stance dynamics of the lossy SLIP model. However, in order to complete the apex return map, we still need to solve for the times and states of bottom and liftoff events.

\subsection{Solving for transition states: bottom and liftoff}

The bottom of stance is reached with the leg at its maximal compression with $\dot{\rho}\left(t_{b}\right)=0$. Using (34), we have

$t_{b}=\left(\pi / 2-\phi-\phi_{2}\right) / \omega_{d}$.

In contrast, liftoff occurs when the toe loses contact with the ground. For a lossless SLIP with $\xi=0$, this corresponds to the usual leg length condition $\rho\left(t_{l o}\right)=\rho_{l o}$, which can easily be solved analytically through the use of (33). However, when damping is present in the system, the liftoff event does not

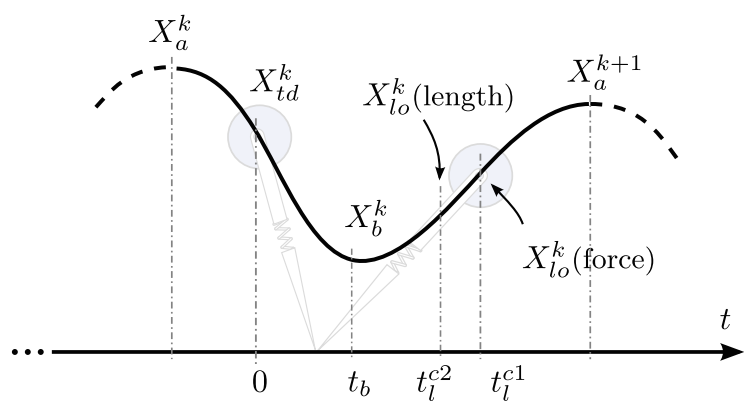

Fig. 3 An illustration of events throughout stance, together with the possibility of two different liftoff conditions, based on either the force condition (39) or the length condition (40)

depend on the leg length alone, but must take into account the ground reaction force on the toe. This can be formalized as a condition on the leg force with

$\kappa\left(1-\rho\left(t_{l}^{c 1}\right)\right)-c \dot{\rho}\left(t_{l}^{c 1}\right)=0$,

which corresponds to the point of vanishing net force exerted on the toe by the spring-damper pair. An alternative liftoff condition arises within platforms where the liftoff leg length can be explicitly chosen by a controller (e.g. as in the Bow-Leg hopper [37]). In such cases, the time of liftoff is given by the solution to the equation

$\rho\left(t_{l}^{c 2}\right)=\rho_{l}$.

Using both (39) and (40), the actual liftoff time can then be found as $t_{l}=\min \left(t_{l}^{c 1}, t_{l}^{c 2}\right)$, with the earlier one of the two events triggering the actual liftoff. Figure 3 illustrates transition events during stance, together with the possible presence of two different liftoff conditions.

Unfortunately, exact analytical solution of these equations is not possible. Even though numerical methods are feasible due to the simple, one-dimensional nature of these equations, we use a sufficiently accurate approximation to compute both liftoff times in order to preserve the analytical nature of our approximations. To this end, we propose a new approximation for the exponential term in (33) with its value at a specific instant during decompression as $e^{-\xi \hat{\omega}_{0} t} \approx e^{-\xi \hat{\omega}_{0} \gamma t_{b}}$, with $\gamma \geq 1$ introduced as a tunable parameter. A reasonable choice is $\gamma=2$, corresponding to compres- 
sion and decompression phases of roughly equal duration. We hence obtain

$$
\begin{aligned}
t_{l}^{c 1} \approx & \left(2 \pi-\arccos \left(\kappa\left(1-F / \hat{\omega}_{0}^{2}\right) /\left(\bar{M} M e^{-\xi \hat{\omega}_{0} \gamma t_{b}}\right)\right)\right. \\
& \left.-\phi-\phi_{3}\right) / \omega_{d}, \\
t_{l}^{c 2} \approx & \left(2 \pi-\arccos \left(\left(\rho_{l}-F / \hat{\omega}_{0}^{2}\right) /\left(M e^{-\xi \hat{\omega}_{0} \gamma t_{b}}\right)\right)\right. \\
& -\phi) / \omega_{d},
\end{aligned}
$$

where we define

$$
\begin{aligned}
\bar{M} & :=\sqrt{\left(c \hat{\omega}_{0}\right)^{2}+\kappa^{2}-2 \kappa c \hat{\omega}_{0} \cos \left(\phi_{2}\right)}, \\
\phi_{3} & :=\arctan \left(\frac{c \hat{\omega}_{0} \sin \left(\phi_{2}\right)}{c \hat{\omega}_{0} \cos \left(\phi_{2}\right)-\kappa}\right) .
\end{aligned}
$$

Once the time instants associated with each event are identified, the corresponding states can be computed, completing all necessary components in the apex return map.

\subsection{Compensating for the effects of gravity}

In this section, we extend the method we introduced in Sect. 3.1 with an explicit correction on the angular momentum to account for the effect of gravity for non-symmetric trajectories, yielding a much larger domain of validity for the resulting analytic approximations.

As illustrated in Fig. 4, angular momenta at touchdown and liftoff are identical only for perfectly symmetric SLIP trajectories, observed only for steadystate running on flat terrain. Unfortunately, for legged robots negotiating rough terrain, non-symmetric trajectories as a result will dominate with deteriorated controller performance.

In the presence of gravity, the instantaneous angular momentum around the toe during stance can be computed as

$p_{\theta}(t)=p_{\theta}(0)+\int_{0}^{t} \rho(\eta) \sin \theta(\eta) d \eta$,

where $p_{\theta}(0)$ denotes the angular momentum at touchdown. We propose a new method to modify our approximations to take into account the total effect of gravity on the angular momentum during stance by a constant average value computed between touchdown and liftoff as

$\hat{p_{\theta}}:=\frac{1}{t_{l o}} \int_{0}^{t_{l o}} p_{\theta}(\eta) d \eta$.

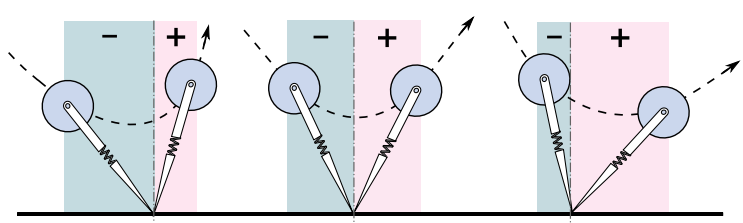

(a)

(b)

(c)

Fig. 4 The total effect of gravity on the magnitude of the angular momentum during stance is (a) negative, (b) zero and (c) positive. Blue and red regions, marked with - and + , represent instantaneous decreasing and increasing effects of gravity on the magnitude of the angular momentum, respectively. Locomotion direction is to the right

Once computed, we could replace all occurrences of $p_{\theta}$ in the derivations of Sect. 3.1 with $\hat{p_{\theta}}$, yielding an analytic correction scheme to compensate for gravitational effects.

Unfortunately, even with the solutions of (33) and (37), exact computation of this expression in closed form is not feasible. Consequently, we propose a new approximation to the integrand in (45), $\tau(t):=$ $\rho(t) \sin \theta(t)$ with an average of its extreme values at touchdown and liftoff as

$\tau(t) \approx \hat{\tau}(t):=\left(\tau(0)+\tau\left(t_{l o}\right)\right) / 2$.

It hence becomes possible to compute an approximate adjustment for the angular momentum of (46) as

$\hat{p_{\theta}}=p_{\theta}(0)+\frac{t_{l o}}{4}\left(\rho(0) \sin \theta(0)+\rho\left(t_{l o}\right) \sin \theta\left(t_{l o}\right)\right)$.

We use this adjusted angular momentum in the "gravity-corrected" performance results presented in Sect. 4.2. Note that computation of (48) requires an initial estimate of system states at liftoff. We use the uncompensated map for this purpose, with the correction incorporated as a second step. This also gives an "iterative" character to our correction method, similarly to the approach adopted in [36].

Our experiments also showed that a final, energybased correction to the stance map significantly increases the accuracy of the resulting approximations. In previous work [14], this correction was based on the fact that the system under study was conservative. In our case, however, damping losses need to be taken into account if the predicted liftoff states are to be corrected. Fortunately, we can use our approximations to 
estimate damping losses as

$$
\begin{aligned}
E_{c}:= & \int_{0}^{t_{l}} c \dot{\rho}^{2}(t) d t \\
= & \frac{1}{2} M^{2} \hat{\omega}_{0}^{2}\left(\xi \left(\cos \left(2 \phi+\phi_{2}\right)+1-e^{-2 \xi \hat{\omega}_{0} t_{l}}\right.\right. \\
& \left.\left.-\cos \left(2 \omega_{d} t_{l}+2 \phi+\phi_{2}\right) e^{-2 \xi \hat{\omega}_{0} t_{l}}\right)\right),
\end{aligned}
$$

which can then be used to compute a corrected liftoff velocity and an associated angular velocity as

$$
\begin{gathered}
\bar{v}_{l}=\sqrt{2\left(E_{t d}-E_{l o}-E_{c}\right)}, \\
\hat{\dot{\theta}}_{l o}=\operatorname{sgn}\left(\dot{\theta}_{l o}\right) \frac{\sqrt{\bar{v}_{l}^{2}-\dot{\rho}_{l o}^{2}}}{\rho_{l o}},
\end{gathered}
$$

with $E_{t d}:=\left(v_{t d}^{2}+\kappa\left(\rho_{t d}-1\right)^{2}+\rho_{t d} \cos \theta_{t d}\right) / 2$ and $E_{l o}:=\left(\kappa\left(\rho_{l o}-1\right)^{2}+\rho_{l o} \cos \theta_{l o}\right) / 2$.

\section{Characterization of predictive performance}

\subsection{Simulation environment and performance criteria}

In the following sections, we investigate the singlestride predictive performance of our approximations to the apex return map under a wide range of initial conditions and control inputs, using normalized percentage errors in different state components. In particular, errors in the apex position and liftoff velocity predictions are respectively defined as

$$
\begin{aligned}
& P E_{a p}:=100 \frac{\left\|\left[y_{a}, z_{a}\right]-\left[\hat{y}_{a}, \hat{z}_{a}\right]\right\|_{2}}{\left\|\left[y_{a}, z_{a}\right]\right\|_{2}}, \\
& P E_{l o v}:=100 \frac{\left\|\left[\dot{\rho}_{l o}, \dot{\theta}_{l o}\right]-\left[\hat{\dot{\rho}}_{l o}, \hat{\dot{\theta}}_{l o}\right]\right\|_{2}}{\left\|\left[\dot{\rho}_{l o}, \dot{\theta}_{l o}\right]\right\|_{2}},
\end{aligned}
$$

where $\left[\hat{y}_{a}, \hat{z}_{a}\right]$ and $\left[\hat{\dot{\rho}}_{l o}, \hat{\dot{\theta}}_{l o}\right]$ denote apex and liftoff states predicted by one of three approximations described earlier, while $\left[y_{a}, z_{a}\right]$ and $\left[\dot{\rho}_{l o}, \dot{\theta}_{l o}\right]$ are obtained by numerical integration of the SLIP model for a single stride. We use the velocity at liftoff rather than the apex to ensure that normalization is meaningful even for non-symmetric gaits with possibly zero apex velocities. Our simulations cover a total of four different dimensions of initial states and control inputs: the apex height $\left(z_{a}\right)$, the apex velocity $\left(\dot{y}_{a}\right)$, the
Table 2 Ranges of initial conditions and control inputs for simulation experiments in dimensionless units

\begin{tabular}{lllll}
\hline$z_{a}$ & $\dot{y}_{a}$ & $\theta_{t d, \text { rel }}$ & $\kappa$ & $\zeta$ \\
\hline$[1.15,1.75]$ & {$[0,2.5]$} & {$[-0.15,0.25]$} & {$[25,200]$} & {$[0,0.4]$} \\
\hline
\end{tabular}

spring constant $(\kappa)$ and the "relative touchdown angle," which we define as

$\theta_{t d, \text { rel }}:=\theta_{t d}-\theta_{t d, n}$,

where $\theta_{t d, n}$ denotes the "neutral" touchdown angle that results in a symmetric SLIP trajectory for the lossless model, defined as the fixed point of the apex return map with $X_{a}={ }_{a}^{a} \mathbf{f}_{\left[\theta_{t d}\right]}\left(X_{a}\right)$ for a given initial apex state $X_{a}$.

The ranges considered for these dimensions were chosen to be consistent with biomechanics literature as well as existing legged robots. In particular, experiments on humans (with $80 \mathrm{~kg}$ mass and $1 \mathrm{~m}$ leg length on average) running at different speeds (in the range of $2.5-6.5 \mathrm{~m} / \mathrm{s}$ ) reveal leg stiffness in the range $[10,50] \mathrm{kN} / \mathrm{m}[5]$. In the robotic domain, the RHex hexapod has an approximate mass of $10 \mathrm{~kg}$, leg length of $0.25 \mathrm{~m}$ and compliant legs with stiffness of around $2000 \mathrm{~N} / \mathrm{m}$ for each leg [32]. Motivated by these observations, Table 2 shows ranges of initial conditions and control inputs we use for our simulations, with the damping ratio, defined as $\zeta:=c /(2 \sqrt{\kappa})$, parameterizing differing amounts of damping for the results of subsequent sections.

For each of our simulations, we check whether the trajectories satisfy two conditions to ensure that we can support meaningful comparisons to existing studies. First, stance trajectories that either never leave the ground $\left(\dot{z}_{l o}<0\right)$ or prevent foot protraction $\left(z_{a}<1\right)$, are excluded. Second, we restrict the maximum allowable leg compression to $25 \%$ of the rest length, excluding trajectories that violate this condition. In each case, we define and compute "ground truth" as the numerical integration of SLIP dynamics for a single stride within MATLAB using a variable time-step, fourth order Runge-Kutta integrator. We then compute estimates of the apex states based on Geyer's and Schwind's approximation methods and our proposed method and compare estimation performances using the error criteria defined above. For the Schwind approximations, we use the 10th iterate (after which further iterations yield no improvements) to make sure we 
obtain the best possible performance for their method. Note that a characterization of performance over a single step is also an accurate indicator of performance across multiple steps since prediction errors accumulate additively if apex states remain in the range of validity for assumptions underlying each method.

\subsection{Performance for non-symmetric, lossless steps}

In this section, we compare the predictive performance of our gravity correction scheme, described in Sect. 3.3, with Geyer's and Schwind's analytic approximations. In order to isolate performance gains resulting from the gravity correction method alone, we use a lossless plant model with $c=0$ for the results of this section. As we will show in the next section, the presence of damping represents a major deviation from the assumptions of Geyer's and Schwind's approximations and makes it the dominant factor in all error measures. Consequently, a fair evaluation of our gravity correction scheme is only possible in the absence of damping.

Figure 5 illustrates mean and standard deviations of percentage prediction errors for all three approximation methods for 192,655 valid simulations out of a total of 257,040 using different initial conditions and control parameters in the ranges shown in Table 2. Corresponding numerical values are listed in the left three columns of Table 3, with most informative entries highlighted in bold. These averaged results show that the proposed gravity corrections result in significant increase in the performance of the approxima- tions, particularly in their prediction of velocity components. This is relatively natural since gravity primarily influences angular momentum and hence the liftoff velocity.

More importantly, however, we expect performance gains resulting from the gravity correction scheme to be much more pronounced for non-symmetric steps. This is also confirmed by our simulations, illustrated in Fig. 6 with plots of mean and standard deviations of liftoff velocity and apex position errors as a function of the relative touchdown angle. Note that by definition, trajectories obtained with $\theta_{t d}$,rel $=0$ are symmetric.

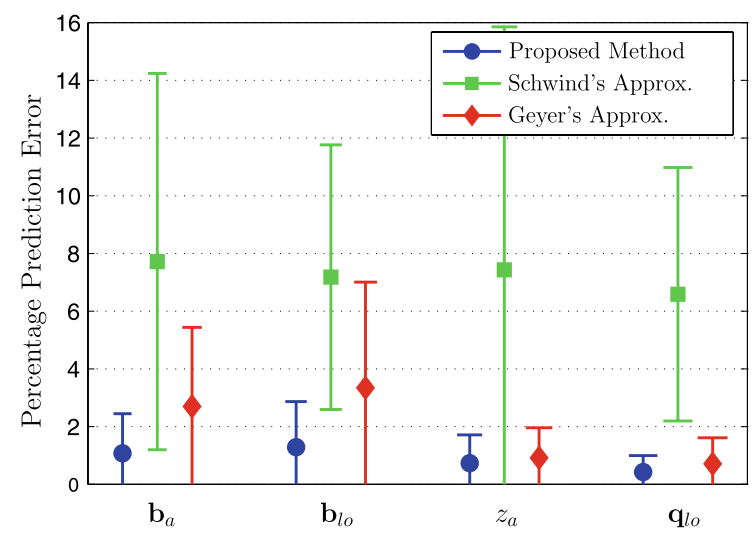

Fig. 5 Percentage prediction errors in apex position $\left(\mathbf{b}_{a}\right)$, liftoff velocity $\left(\dot{\mathbf{b}}_{l o}\right)$, apex height $\left(z_{a}\right)$ and liftoff position $\left(\mathbf{q}_{l o}\right)$ for the proposed method, Schwind's iterative approximations [36] and Geyer's approximations [14] in the absence of damping, but with non-symmetric steps. Mean errors across 192,655 valid simulations, while the vertical bars represent associated standard deviations

Table 3 Percentage prediction errors for Geyer's, Schwind's and our methods in apex position $\left(\mathbf{b}_{a}\right)$, liftoff velocity $\left(\dot{\mathbf{b}}_{l o}\right)$, apex height $\left(z_{a}\right)$, liftoff position $\left(\mathbf{q}_{l o}\right)$, apex energy $\left(E_{a}\right)$ and stance time $\left(t_{s}\right)$. Simulations without and with damping are respectively reported on the left and right sides of the table. In each case, the performance of each method is summarized by the mean, standard deviation and maximum values for their percentage prediction errors across all simulations covering the ranges in Table 2. Most informative entries are highlighted with bold font

\begin{tabular}{|c|c|c|c|c|c|c|c|c|c|c|c|c|}
\hline & \multicolumn{6}{|c|}{ SLIP model without damping } & \multicolumn{6}{|c|}{ SLIP model with damping } \\
\hline & \multicolumn{2}{|c|}{ Geyer's method } & \multicolumn{2}{|c|}{ Schwind's method } & \multicolumn{2}{|c|}{ Proposed method } & \multicolumn{2}{|c|}{ Geyer's method } & \multicolumn{2}{|c|}{ Schwind's method } & \multicolumn{2}{|c|}{ Proposed method } \\
\hline & $\mu \pm \sigma$ & $\max$ & $\mu \pm \sigma$ & $\max$ & $\mu \pm \sigma$ & $\max$ & $\mu \pm \sigma$ & $\max$ & $\mu \pm \sigma$ & $\max$ & $\mu \pm \sigma$ & $\max$ \\
\hline $\mathbf{b}_{a}$ & $2.70 \pm 2.74$ & 27.3 & $7.72 \pm 6.52$ & 51.8 & $1.07 \pm 1.37$ & 18.4 & $53.3 \pm 33.0$ & 221 & $54.7 \pm 33.6$ & 205 & $\mathbf{0 . 7 5} \pm \mathbf{1 . 2 7}$ & 24.2 \\
\hline$\dot{\mathbf{b}}_{l o}$ & $3.34 \pm 3.66$ & 41.3 & $7.18 \pm 4.59$ & 24.5 & $1.29 \pm 1.58$ & 26.3 & $53.2 \pm 41.0$ & 280 & $57.7 \pm 39.1$ & 280 & $1.40 \pm 2.27$ & 46.4 \\
\hline$z_{a}$ & $0.91 \pm 1.04$ & 15.3 & $7.43 \pm 8.42$ & 58.6 & $0.73 \pm 0.98$ & 7.56 & $40.6 \pm 26.7$ & 213 & $49.0 \pm 30.3$ & 206 & $0.42 \pm 0.68$ & 7.55 \\
\hline $\mathbf{b}_{l o}$ & $0.71 \pm 0.90$ & 10.9 & $6.58 \pm 4.39$ & 22.7 & $0.42 \pm 0.57$ & 3.71 & $5.70 \pm 4.70$ & 44.1 & $4.36 \pm 2.48$ & 23.0 & $0.32 \pm 0.49$ & 3.87 \\
\hline$E_{a}$ & $0.00 \pm 0.00$ & 0.00 & $0.00 \pm 0.00$ & 0.00 & $0.00 \pm 0.00$ & 0.00 & $32.7 \pm 20.5$ & 189 & $32.7 \pm 20.5$ & 189 & $0.23 \pm 0.38$ & 5.20 \\
\hline$t_{s}$ & $0.35 \pm 0.47$ & 4.36 & $18.9 \pm 0.30$ & 20.3 & $0.38 \pm 0.48$ & 4.28 & $12.6 \pm 8.08$ & 48.5 & $9.86 \pm 4.84$ & 24.7 & $0.38 \pm 0.52$ & 6.03 \\
\hline
\end{tabular}


Fig. 6 Percentage prediction errors for all three methods for liftoff velocity (left) and apex position (right) as a function of the relative touchdown angle $\theta_{t d \text {,rel }}$. Each data point represents the mean of all valid simulations with the corresponding relative touchdown angle. Standard deviation bars are only shown for the proposed method for clarity

Fig. 7 Percentage prediction errors in liftoff velocity (left) and apex position (right) for all three methods as a function of increasing damping ratio. Error axes are plotted in logarithmic scale to simultaneously show the predictive performances of Schwind's and Geyer's approximations with the proposed method, which yields mean errors that are two orders of magnitude better than its alternatives
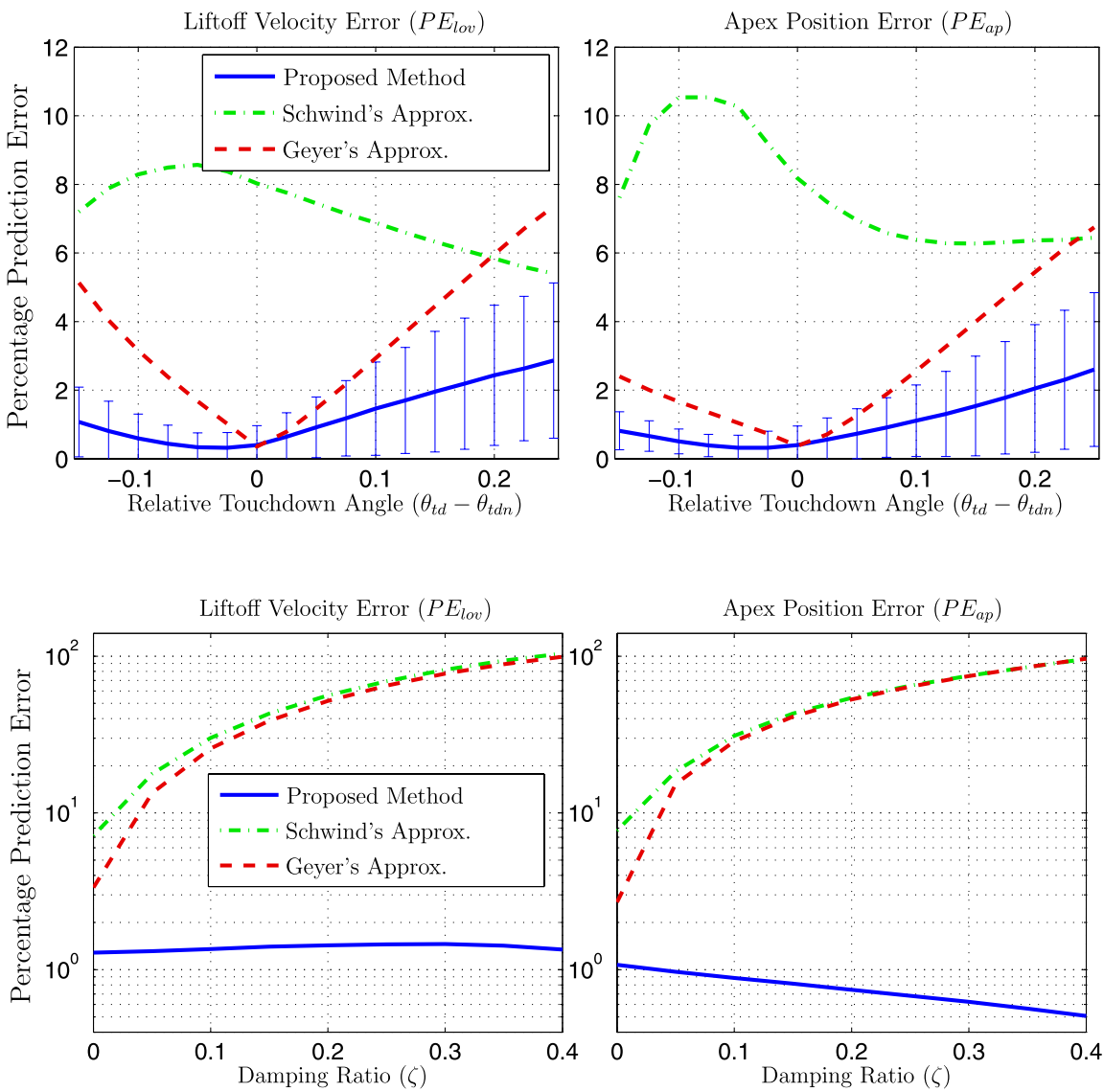

For such steps, our approximation becomes equivalent to Geyer's method as is also evident from the coincident plots in the figure. In contrast, positive and negative values of $\theta_{t d \text {,rel }}$ result in decelerating and accelerating steps, respectively. In both of these ranges, the gravity correction method we propose outperforms existing alternatives, yielding very accurate analytic approximations that can be effectively used for applications such as locomotion on rough terrain that require frequent use of non-symmetric steps.

\subsection{Predictive performance in the presence of damping}

As noted in the previous section, the presence of damping challenges the energy conservation assumption that underlies both Geyer's and Schwind's approximations. The right side of Table 3 illustrates percentage prediction errors for all three methods in the presence of non-negligible damping. As is evident from these error figures, existing analytic approximations for the SLIP model have deteriorated predictive performance (with errors exceeding 50\%), while the proposed method remains equally accurate with errors under $2 \%$. There is even a slight increase in accuracy for our method compared to its performance for the lossless case, which can be attributed to shorter stance times arising from damped radial trajectories.

Figure 7 illustrates the dependence of prediction errors for all three methods on the dimensionless damping ratio $\zeta:=c /(2 \sqrt{\kappa})$, plotted in logarithmic scale so that the trends of all three methods are simultaneously visible. For even small amounts of damping with $\zeta=0.1$, the proposed approximations perform almost two orders of magnitude better than best available alternatives in the literature. As noted above, there is even a slight increase in the prediction performance for the apex position as the amount of damping increases as a result of shorter stance times that bring trajectories closer to satisfying assumptions underlying the derivations of Sect. 3.1. 
Another important performance measure for our approximations would have been the accuracy of its prediction for local linearizations of the return map, often used to analyze stability properties of both openloop and feedback control strategies. Some of our preliminary investigations show that our approximations also remain accurate in this regard. However, we leave the treatment of this topic outside the scope of the present paper since an adequate coverage would substantially lengthen the presentation.

\section{Application: gait control of monopedal running}

\subsection{Deadbeat controller for regulating apex states}

A natural application of an analytically formulated apex return map for the spring-mass hopper is the design of a deadbeat controller to regulate and stabilize the progression of its apex states. The control problem hence consists of finding appropriate control inputs $\mathbf{u}:=\left[\theta_{t d}, \rho_{t d}, \rho_{l o}\right]$ to satisfy

$X_{a}^{*}={ }_{a}^{a} \mathbf{f}\left(X_{a}, \mathbf{u}\right)$,

where $X_{a}$ and $X_{a}^{*}$ denote the current and desired apex states, respectively, and leg spring constants are chosen to be constant with $\kappa_{c}=\kappa_{d}=\kappa$.

Inversion of the associated map, however, still involves three coupled variables. We start by observing that we are primarily interested in sustained, steadystate locomotion so the cyclic variable $y_{a}$ can comfortably be eliminated from the domain of the controller, leaving only the apex height $z_{a}$ and the apex speed $\dot{y}_{a}$ as variables of interest. However, the solution of the resulting equation is not as simple and requires an iterative procedure.

Initially, we assume that no damping is present in the system and solve the energy balance equation

$$
\begin{gathered}
\kappa\left(\rho_{t d}-1\right)^{2}-\kappa\left(\rho_{l o}-1\right)^{2} \\
=E\left(z_{a}^{*}, \dot{y}_{a}^{*}\right)-E\left(z_{a}, \dot{y}_{a}\right)
\end{gathered}
$$

for the control inputs $\rho_{t d}$ and $\rho_{l o}$, noting that either $\rho_{t d}=1$ or $\rho_{l o}=1$ (i.e. equal to the leg rest length in dimensionless units) when the desired energy differential is negative or positive, respectively. Once these control inputs are determined, (55) reduces to a onedimensional equation whose solution can be formulated as a minimization problem with

$\theta_{t d}=\underset{\frac{-\pi}{2}<\theta<\frac{-\pi}{2}}{\operatorname{argmin}}\left(\dot{y}_{a}^{*}\left(\pi_{\dot{\bar{y}}_{a}} \circ{ }_{a}^{a} \mathbf{f}\left(X_{a}, \theta, \rho_{t d}, \rho_{l o}\right)\right)\right)^{2}$,

and whose numerical solution is feasible due to its one-dimensional and monotonic nature. Having computed estimates of all control inputs for a lossless system, we can now estimate damping losses using (49) and solve the complete energy balance equation

$$
\begin{aligned}
& \kappa\left(\rho_{t d}-1\right)^{2}-\kappa\left(\rho_{l o}-1\right)^{2} \\
& \quad=E\left(z_{a}^{*}, \dot{y}_{a}^{*}\right)-E\left(z_{a}, \dot{y}_{a}\right)+E_{c}
\end{aligned}
$$

to yield better estimates of the control inputs $\rho_{t d}$ and $\rho_{l o}$, as before. Using these new estimates, we can obtain a new solution for the touchdown angle through (57), which now takes into account damping losses as well. This results in an effective one-stride deadbeat controller for the regulation of apex height and horizontal speed. Note that (58) and (57) can be iteratively applied to obtain increasingly accurate solutions for the control inputs.

\subsection{Steady-state tracking performance}

In order to show that our analytic approximations provide a good basis for the design of high-performance gait controllers, we compare the steady-state tracking performance of the controller described in Sect. 5.1 to similar designs based on Geyer's and Schwind's approximations. Controllers based on Schwind's approximations are rather simple with no consideration of damping and have been previously presented in the literature [9, 29, 31]. Deadbeat control based on Geyer's approximations closely parallels the descriptions of Sect. 5.1 except for the iterative treatment of damping. We omit detailed derivations for controllers associated with these two methods for space considerations.

In order to obtain a comprehensive picture for the performance of all three controllers, we ran simulations with the SLIP models with different spring constants $\kappa \in[25,200]$ and damping coefficients $\zeta \in$ $[0,0.4]$, with a wide range of apex state goals in $z_{a}^{*} \in$ $[1.3,1.6]$ and $\dot{y}_{a}^{*} \in[0.5,2.25]$. For each goal, simulations were started from a range of different initial conditions around the goal with $z_{a} \in\left[z_{a}^{*}-0.15, z_{a}^{*}+0.15\right]$ 


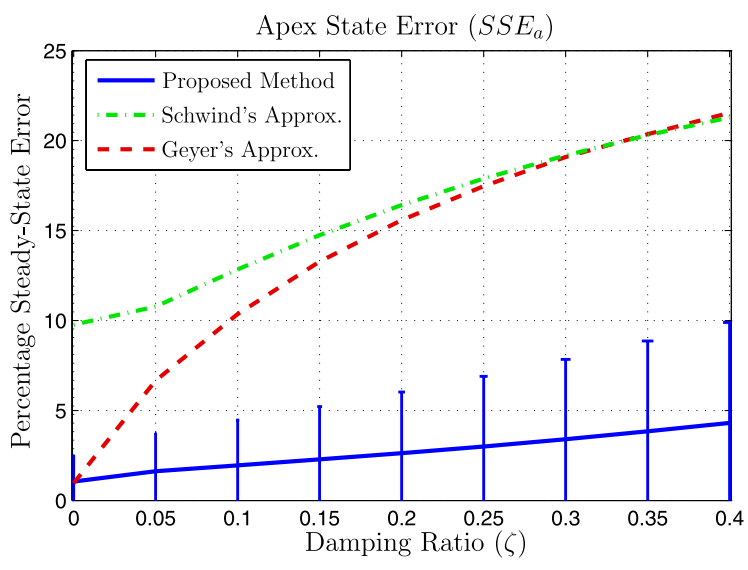

Fig. 8 Percentage steady-state errors in the norm of the non-dimensional apex state vector for all three methods as a function of the damping ratio $\zeta$. Each data point represents the mean of all valid simulations with the corresponding damping ratio. Standard deviation bars are only shown for the proposed method for clarity

and $\dot{y}_{a} \in\left[\dot{y}_{a}^{*}-0.25, \dot{y}_{a}^{*}+0.25\right]$. In each case, simulations were run using each one of three controllers for eight steps, at the end of which convergence to steady state was confirmed with a tolerance of $10^{-4}$ and the difference from the desired goal was measured. In particular, we are interested in the percentage error in non-cyclic components of the apex state, defined as

$S S E_{a}:=100 \frac{\left\|\left[z_{a}, \dot{y}_{a}\right]-\left[z_{a}^{*}, \dot{y}_{a}^{*}\right]\right\|_{2}}{\left\|\left[z_{a}^{*}, \dot{y}_{a}^{*}\right]\right\|_{2}}$.

Note that this error measure incorporates both the apex height and speed in dimensionless coordinates, and avoids normalization problems associated with a vanishing apex velocity.

Figure 8 illustrates mean percentage tracking errors in the apex state at steady state, $S S E_{a}$, for all three methods as a function of the damping ratio. The gait controller design based on our approximations significantly improves the performance of other methods, with average steady-state errors consistently below $4 \%$. Note that deadbeat control based on Geyer's approximations has identical performance to ours in the absence of damping since steady-state locomotion consists of symmetric steps for the lossless SLIP [35]. Nevertheless, increasing amounts of damping result in substantial deterioration of controllers based on both Geyer's and Schwind's methods since the resulting energy losses dominate the associated prediction errors.

\section{Conclusion}

In this paper, we introduced a simple yet accurate new analytical approximation to the stance trajectories of a dissipative Spring-Loaded Inverted Pendulum model with linear leg compliance. Conservative versions of this model were shown to be very successful in describing center of mass motions of running animals with widely different sizes and morphologies. However, existing literature on this model almost universally excludes dissipative effects, and exclusively focuses on symmetric steps that occur during locomotion at steady state. These two limitations substantially impair their applicability in the design and control of legged robots on rough terrain, where damping is inevitable and significant, and non-symmetric steps are frequent.

We have presented extensive simulation results, covering a large range of operating conditions and parameter settings within a dimensionless formulation to show that our approximate map can provide extremely accurate estimates for the trajectories of the dissipative SLIP model, with errors that are consistently below $2 \%$ for all but the most extreme conditions. Not only does our method by far outperform available alternatives in the literature in the presence of damping (with up to two orders of magnitude improvement in predictive accuracy), but it also shows improved performance on the lossless SLIP model for non-symmetric steps owing to a novel gravity correction method also introduced in this paper. Overall, the methods we present in this paper provide the currently most accurate closed-form approximations to the otherwise nonintegrable trajectories of the dissipative SLIP model, whose importance in both the modeling and control of legged locomotion has long been established.

In addition to our systematic characterization of the predictive performance of our approximations, we have also demonstrated their utility in the context of a gait controller for the dissipative SLIP model. The simple analytic form of our approximations provides a very straightforward way in which a deadbeat stride controller can be formulated, naturally taking damping induced energy losses into account and hence substantially improving the performance of similar control strategies in the literature. Once again, through a systematic set of simulations, we show that the resulting feedback controller is capable of regulating gait parameters of steady-state running with tracking errors consistently below $4 \%$, almost an order of magni- 
tude better than other methods for a dissipative SLIP model.

Acknowledgements Ömür Arslan was supported by the Department of Electric and Electronics Engineering, Bilkent University. M. Mert Ankaralı was supported by the National Scientific and Technological Council of Turkey (TUBITAK).

\section{References}

1. Ahmadi, M., Buehler, M.: Controlled passive dynamic running experiments with the ARL-monopod II. IEEE Trans. Robot. 22(5), 974-986 (2006)

2. Alexander, R.M.: Three uses for springs in legged locomotion. Int. J. Robot. Res. 9(2), 53-61 (1990)

3. Alexander, R.M., Jayes, A.S.: Vertical movement in walking and running. J. Zoology, Lond. 185, 27-40 (1978)

4. Altendorfer, R., Koditschek, D.E., Holmes, P.: Stability analysis of legged locomotion models by symmetryfactored return maps. Int. J. Robot. Res. 23(10-11), 979999 (2004)

5. Arampatzis, A., Briggemann, G.-P., Metzler, V.: The effect of speed on leg stiffness and joint kinematics in human running. J. Biomech. 32, 1349-1353 (1999)

6. Arslan, O.: Model based methods for the control and planning of running robots. M.Sc., Bilkent Univ., Ankara, Turkey, July (2009)

7. Arslan, O., Saranli, U., Morgul, O.: Reactive footstep planning for a planar spring-mass hopper. In: Proceedings of the International Conference on Intelligent Robots and Systems, St. Louis, MO, October (2009)

8. Blickhan, R., Full, R.J.: Similarity in multilegged locomotion: bouncing like a monopode. J. Comp. Physiol. A Neuroethol. Sens. Neural Behav. Physiol. 173(5), 509-517 (1993)

9. Carver, S.: Control of a spring-mass hopper. Ph.D., Cornell University, January (2003)

10. Cham, J.G., Karpick, J.K., Cutkosky, M.R.: Stride period adaptation of a biomimetic running hexapod. Int. J. Robot. Res. 23(2), 141-153 (2004)

11. Diamond, J.: Why animals run on legs, not on wheels. Discover 4(9), 64-67 (1983)

12. Farley, C.T., Ferris, D.P.: Biomechanics of walking and running: center of mass movements to muscle action. Exerc. Sport Sci. Rev. 26, 253-283 (1998)

13. Full, R.J., Koditschek, D.E.: Templates and anchors: neuromechanical hypotheses of legged locomotion. J. Exp. Biol. 202, 3325-3332 (1999)

14. Geyer, H., Seyfarth, A., Blickhan, R.: Spring-mass running: simple approximate solution and application to gait stability. J. Theor. Biol. 232(3), 315-328 (2005)

15. Gregorio, P., Ahmadi, M., Buehler, M.: Design, control, and energetics of an electrically actuated legged robot. IEEE Trans. Syst. Man Cybern. 27(4), 626-634 (1997)

16. Grimmer, S., Ernst, M., Gunther, M., Blickhan, R.: Running on uneven ground: leg adjustment to vertical steps and self-stability. J. Exp. Biol. 211(18), 2989-3000 (2008)

17. Holmes, P.: Poincaré, celestial mechanics, dynamicalsystems theory and "chaos". Phys. Rep. (Review Section of Physics Letters), 193, 137-163 (1990)
18. Holmes, P., Full, R., Koditschek, D., Guckenheimer, J.: The dynamics of legged locomotion: models, analyses, and challenges. SIAM Rev. 48(2), 207-304 (2006)

19. Hurst, J.W., Chestnutt, J.E., Rizzi, A.A.: Design and philosophy of the BiMASC, a highly dynamic biped. In: Proceedings of the International Conference on Robotics and Automation, pp. 1863-1868, April 10-14, Roma (2007)

20. Jindrich, D.L., Qiao, M.: Maneuvers during legged locomotion. Chaos 19(2), 026105 (2009)

21. Koditschek, D.E., Buehler, M.: Analysis of a simplified hopping robot. Int. J. Robot. Res. 10(6), 587-605 (1991)

22. LaBarbera, M.: Why the wheels won't go. Am. Nat. 121(3), 395-408 (1983)

23. Nelson, G.M., Quinn, R.D.: Posture control of a cockroachlike robot. IEEE Control Syst. Mag. 19(2), 9-14 (1999)

24. Playter, R., Buehler, M., Raibert, M.: BigDog. Proc. SPIE 6230 (2006). doi: $10.1117 / 12.084087$

25. Poulakakis, I., Grizzle, J.W.: Formal embedding of the spring loaded inverted pendulum in an asymmetric hopper. In: Proceedings of the European Control Conference, Kos (2007)

26. Poulakakis, I., Papadopoulos, E., Buehler, M.: On the stability of the passive dynamics of quadrupedal running with a bounding gait. Int. J. Robot. Res. 25(7), 669-687 (2006)

27. Pratt, J., Pratt, G.: Intuitive control of a planar bipedal walking robot. In: Proceedings of the International Conference on Robotics and Automation, pp. 2014-2021, Leuven, Belgium (1998)

28. Raibert, M.: Legged Robots that Balance. MIT Press Series in Artificial Intelligence. MIT Press, Boston (1986)

29. Saranli, U.: Dynamic locomotion with a hexapod robot. Ph.D., The University of Michigan, Ann Arbor, MI, September (2002)

30. Saranli, U., Koditschek, D.E.: Template based control of hexapedal running. In: Proceedings of the IEEE International Conference on Robotics and Automation, vol. 1, pp. 1374-1379, Taipei, Taiwan, September (2003)

31. Saranli, U., Schwind, W.J., Koditschek, D.E.: Toward the control of a multi-jointed, monoped runner. In: Proceedings of the IEEE International Conference on Robotics and Automation, vol. 3, pp. 2676-2682, New York (1998)

32. Saranli, U., Buehler, M., Koditschek, D.E.: RHex: a simple and highly mobile robot. Int. J. Robot. Res. 20(7), 616-631 (2001)

33. Sato, A., Buehler, M.: A planar hopping robot with one actuator: design, simulation, and experimental results. In: Proceedings of the International Conference on Intelligent Robots and Systems, vol. 4, pp. 3540-3545, Sept.-2 Oct., Sendai (2004)

34. Schmitt, J.: A simple stabilizing control for sagittal plane locomotion. J. Comput. Nonlinear Dyn. 1(4), 348-357 (2006)

35. Schwind, W.J.: Spring loaded inverted pendulum running: a plant model. Ph.D., University of Michigan, Ann Arbor (1998)

36. Schwind, W.J., Koditschek, D.E.: Approximating the stance map of a 2 DOF monoped runner. J. Nonlinear Sci. 10(5), 533-568 (2000)

37. Zeglin, G.: The Bow Leg hopping robot. Ph.D., Carnegie Mellon University, Pittsburgh, CMU-RI-TR-99-33, October (1999) 